\title{
Start stopping smartly
}

\author{
Fiona Godlee editor in chief
}

The BMJ

There can be little that's more central to a doctor's role than clearly communicating the benefits and harms of treatments. Yet it's one of the hardest things to get right, and it seems to be getting harder. Approaches once considered adequate no longer meet the needs of shared decision making or the complexity of the evidence base. At a meeting last week at the Academy of Medical Sciences delegates agreed that doctors, patients, journalists, and the public often struggled to grasp numerical information, creating ample scope for miscommunication and bedevilling efforts to achieve truly shared and personalised decisions.

The meeting was part of the academy's wider ongoing inquiry into how we can all best use evidence to judge the benefits and harms of treatments, which was prompted by concerns about loss of trust in research and medicine (doi:10.1136/bmj.h3300), with a specific focus on several $B M J$ investigations and campaigns: tamiflu, statins, and conflicts of interest (www.bmj. com/campaigns). Delegates concluded that equipping people to evaluate claims about treatments should start in schools; that numbers, words, and pictures were all necessary to cater for different situations and individuals; that doctors needed to communicate uncertainty where evidence was unclear or disputed; that patients differ in their attitudes to taking pills, whatever the evidence indicates; and that information should be used to inform rather than persuade.

We need shared decision making when starting treatments, of course, but also when stopping them, as Jesse Jansen and colleagues explain this week (doi:10.1136/bmj.i2893). Their review of the literature on "deprescribing" focuses on older people, who may find it hardest to understand the evidence and are most at risk from the ill effects of polypharmacy. Their article is full of intriguing insights into human nature, including that we are more willing to risk harms arising from inaction than from action, and that, paradoxically, once people are taking a drug, continuing it unchanged is perceived as inaction while stopping it is perceived as an action. The first of their proposed four steps is to make patients aware that stopping treatments is even an option. Then comes the discussion of benefits and harms, an exploration of the patient's preferences, and the decision itself.

Where are doctors to find the time for these health and life improving conversations? The authors suggest protected time, dedicated resources, and even specific remuneration for medicine reviews. The savings in drug costs, reduced adverse effects, and improved quality at the end of life could be worth having. 